\title{
Behavior Modeling for a Beacon-Based Indoor Location System
}

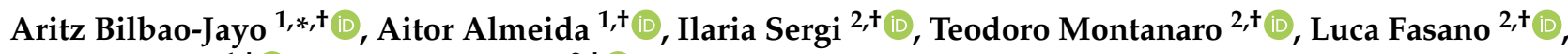 \\ Mikel Emaldi ${ }^{1,+}+$ and Luigi Patrono ${ }^{2,+}(\mathbb{\infty})$
}

1 DeustoTech Institute of Technology, University of Deusto, Av. Universidades 24, 48007 Bilbao, Spain; aitor.almeida@deusto.es (A.A.); m.emaldi@deusto.es (M.E.)

2 Department of Engineering for Innovation, University of Salento, Via Monteroni snc, 73100 Lecce, Italy; ilaria.sergi@unisalento.it (I.S.); teodoro.montanaro@unisalento.it (T.M.);

luca.fasano@studenti.unisalento.it (L.F.); luigi.patrono@unisalento.it (L.P.)

* Correspondence: aritzbilbao@deusto.es; Tel.: +34-944-139-000

+ These authors contributed equally to this work.

Citation: Bilbao-Jayo, A.; Almeida,

A.; Sergi, I.; Montanaro, T.; Fasano, L.; Emaldi, M.; Patrono, L. Behavior Modeling for a Beacon-Based Indoor Location System. Sensors 2021, 21, 4839. https://doi.org/10.3390/ s21144839

Academic Editor: Joanna Kolodziej

Received: 14 June 2021

Accepted: 13 July 2021

Published: 15 July 2021

Publisher's Note: MDPI stays neutral with regard to jurisdictional claims in published maps and institutional affiliations.

Copyright: (c) 2021 by the authors. Licensee MDPI, Basel, Switzerland. This article is an open access article distributed under the terms and conditions of the Creative Commons Attribution (CC BY) license (https:/ / creativecommons.org/licenses/by/ $4.0 /)$.

\begin{abstract}
In this work we performed a comparison between two different approaches to track a person in indoor environments using a locating system based on BLE technology with a smartphone and a smartwatch as monitoring devices. To do so, we provide the system architecture we designed and describe how the different elements of the proposed system interact with each other. Moreover, we have evaluated the system's performance by computing the mean percentage error in the detection of the indoor position. Finally, we present a novel location prediction system based on neural embeddings, and a soft-attention mechanism, which is able to predict user's next location with $67 \%$ accuracy.
\end{abstract}

Keywords: ambient assisted living; indoor positioning; internet of things; performance; smartphone; smartwatch; wearable device

\section{Introduction}

The advances in hardware and software technologies have led to the adoption of smartenvironments in many contexts of our daily lives. Smart homes and smart buildings are already equipped with a multitude of embedded devices, along with connected sensors and actuators [1]. Several real cases already exemplify smart cities, which use the opportunities provided by innovative technologies to improve the lives of their inhabitants [2]. In such settings, smart environments are expected to play a crucial role for coping with the needs of sustainability, energy distribution, mobility, health and public safety/security [3]. A particular focus is the realization of ambient assisted living (AAL) solutions to enable elderly people to live independently for as long as possible, without intrusiveness from others. These solutions benefit from Internet of Things (IoT)-enabling technologies to improve elderly life thanks to the introduction of intelligent, connected devices [4].

Several AAL applications have been developed that have user positioning as their core capability. Elderly care [5], guidance systems [6], energy consumption [7] and security [8] are only some of the possible applications of indoor positioning information. Based on indoor positioning, it is possible to identify where a user is located and to predict his/her future locations based on the recent location history. In this paper, the indoor positioning issue is addressed by considering the performance obtained while using two different kinds of device to estimate the indoor position: a smartphone and a smartwatch. With both devices, the Bluetooth Low Energy (BLE) technology was exploited to obtain indoor positioning information. A generic home has been equipped with BLE beacon infrastructure, and several tests have been carried out with different configurations in terms of the number and models of beacons in each room. For each test campaign, the performance in terms of mean percentage error in the detection of the indoor position was calculated using a smartphone and a smartwatch, and the results have been discussed. 
For the location prediction, we present an algorithm based on using neural embeddings to represent the locations of a house and an attention-based mechanism that instead of being applied to the hidden states of the neural network architecture is used to modify those embeddings.

The rest of the paper is structured as follows. Section 2 contains an analysis of the state of the art. In Section 3 we describe the overall architecture of the system and in Section 4 the location prediction algorithm. Section 5 contains an explanation of the testing environment and we discuss the results of the experiments in Section 6. Finally, in Section 7 we draw the conclusions and propose future areas of research.

\section{Related Work}

\subsection{Indoor Location}

Indoor positioning systems (IPS) are an essential part of any intelligent environment or pervasive computing system. Indoor positioning has been used to model users' behavior in order to detect early risks related to frailty in elders [9], guide museum visits [10] and coordinate emergency responses [11]. There are different approaches and technologies that have been proposed over the years to tackle indoor positioning. Vision-based approaches use either visible light systems [12] or infrared signals, such as the Active Badge Location System, wherein a wearable tag emits an infrared code that is captured by an interconnected network of sensors [13]. Other vision-based systems use computer vision to detect specifically generated bidimensional codes in order to locate users and devices in an intelligent environment, such as the TRIP location system [14]. In the context of ambient assisted living, in [15] a video-based monitoring system for elderly care was proposed. The main objectives of this system are to preserve elderly independence and increase the efficiency of the homecare practices. The main disadvantage of the vision-based technology lies in the cost, which is still too high, especially for systems with very high precision. Alternatives to these systems are the radio frequency-based systems, such as those using Wi-Fi [16], RFID or Bluetooth.

Radio frequency identification (RFID) is one of the most popular wireless technologies for tracing and positioning $[17,18]$. The main advantage of this technology is the capability to work in the absence of line of sight (LoS). An example of this is the work done in [19]. The authors used Bayesian probability and k-nearest neighbors in combination with RFID tags. Other authors applied a deep belief network as fingerprinting-based RFID indoor localization algorithm [20]. Additionally, a combination of hyperbolic positioning and genetic algorithms has been used in order to compute the phase offset caused by the interference between tags [21]. NFC systems, such as [22], can be considered a sub-category of RFID systems. In most cases, such systems have the drawback of requiring a smartphone to approach deployed beacon. This type of active participation from the users is not desirable in most scenarios.

Bluetooth technology is an alternative for indoor positioning [23]. It can guarantee a low cost since it is integrated in most of our devices that are used daily, such as tablets and smartphones. Moreover, the spread of the emerging BLE technology makes BT also energy efficient, which is a key requirement in many indoor applications. This efficiency allows for higher measuring rates when determining a user's location and for longer battery life. For these reasons, BLE is considered as one of the most suitable positioning technologies for indoor positioning currently. The recent rise of iBeacons by Apple has contributed to the rapid spread of this technology, which is used to provide information and location services [24] in a completely innovative way. The accuracy of BLE for indoor locating has been extensively studied by several authors [25]. Subedi et al. [26] proposed the use of weighted centroid localization alongside the received signal strength indications from the neighboring BLE beacons. However, in order to achieve similar accuracy rates to Wi-Fi based approaches, BLE beacon-based approaches require more beacons than Wi-Fi APs [27]. 
Ultra-Wide Band (UWB) is another alternative for accurate indoor positioning. García et al. [28] presenteded a novel system for indoor positioning using UWB in highly complex environments where there are non-line-of-sight (NLOS) conditions. To do so, the authors used an extender Kalman filter for a NLOS detection algorithm. UWB has been widely applied in the tracking of sports activities in indoor environments [29,30]. A more extensive analysis of the state-of-the-art can be found in the following reviews: [31,32].

\subsection{Behavior Prediction and Modeling}

User behavior prediction and modeling is an area of research applied to several domains. As discussed in [33], behavior prediction is a core problem to be solved in the creation of more energy efficient and sustainable spaces. In [34], the authors applied behavior prediction to the online behavior in order to identify malicious users. In [35] it was used for marketing purposes. Behavior prediction is a commonly used technique in both real and virtual intelligent spaces. In [4], behavior was used to identify the risks related to mild cognitive impairments and frailty in the elderly in IoT-augmented spaces. The authors of [36] used behavior prediction to create more intelligent automated homes. In [37], the authors used similar behavior predicting approaches, but in this case to predict the behavior in virtual spaces.

Different techniques have been used to tackle this problem. Almeida et al. [38,39] have studied the usage of both convolutional neural networks (CNNs) and long shortterm memory (LSTMs) architectures to predict users' behavior while representing actions with neural embeddings. A LSTM approach was also used in [40] to learn and predict design commands based upon building information modeling (BIM) event log data stored in Autodesk Revit journal files. In [41] the authors followed a neuro-fuzzy approach. A Gaussian radial basis function neural network (GRBF-NN) was trained based on the example set generated by a fuzzy rule-based system (FRBS) and the 360-degree feedback of the user. Kim et al. [42] studied using RNN architectures in order to predict multi-domain behavior. In [43], the authors used both attention and memory mechanisms in their neural network architectures to improve the prediction results.

\section{System Architecture}

In Figure 1, the system's overall architecture is depicted. It mainly consists of the following components:

- BLE beacon infrastructure.

- A monitoring device to capture positioning data.

- A cloud server to store and process captured data.

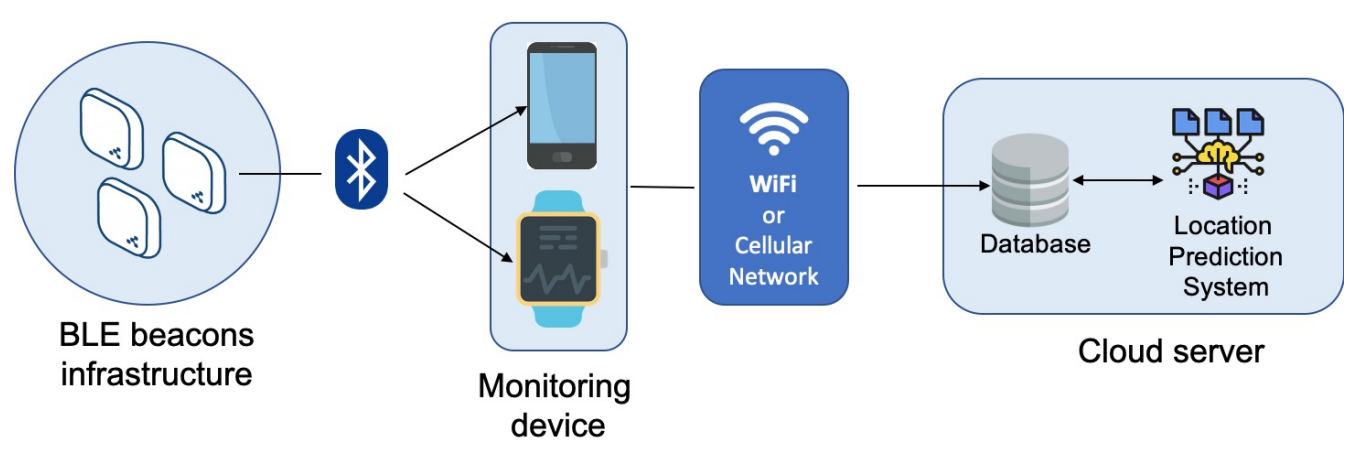

Figure 1. The system's overall architecture.

Beacons are small radio transmitters that send Bluetooth signals. They are available in different sizes and shapes, making them suitable for a wide range of applications and allowing them to be easily integrated into any environment unobtrusively. A beacon is cost-effective and can be installed easily, and its position can be determined with to within a few meters. The BLE standard is also very energy efficient. Beacons can be used in server- 
based (asset tracking) and client-based (indoor navigation) applications. The last option was used in our study. Specifically, in the proposed solution, the indoor environment is equipped with a BLE beacon infrastructure. In particular, a BLE beacon is placed in each room, but in large rooms or long corridors, more beacons can be placed.

On the server side, every association between a beacon (i.e., the MAC address of the beacon) and its location (i.e., the room in which it is located) is stored in the database. When the application starts, this beacon/room map is transmitted from the server to the local database on the monitoring device. In this way, the application performs preliminary filtering during the scanning phase and only considers signals from beacons that are part of the implemented infrastructure for subsequent operations. The monitoring device consists of a smartphone or smartwatch running a specially designed and implemented application. In particular, the mobile application performs repeated Bluetooth scanning in configurable time intervals. With our settings, Bluetooth scanning lasts 10 seconds, and the next scan starts $15 \mathrm{~s}$ after the end of the previous one. During the scanning phase (i.e., within a 10-s interval), each beacon will be detected multiple times, triggering an event. Specifically, the average value of the detected RSSI and the average value of the transmission power (TxPower) (the power at which the beacon broadcasts its signal) are calculated. At the conclusion of the scanning process, a list of beacons identified by MAC address is obtained, along with relative average RSSI and TxPower values. Using these values, the calculateRating function in Listing 1 is applied for each beacon.

Listing 1. Function used to calculate the RSSI accuracy.

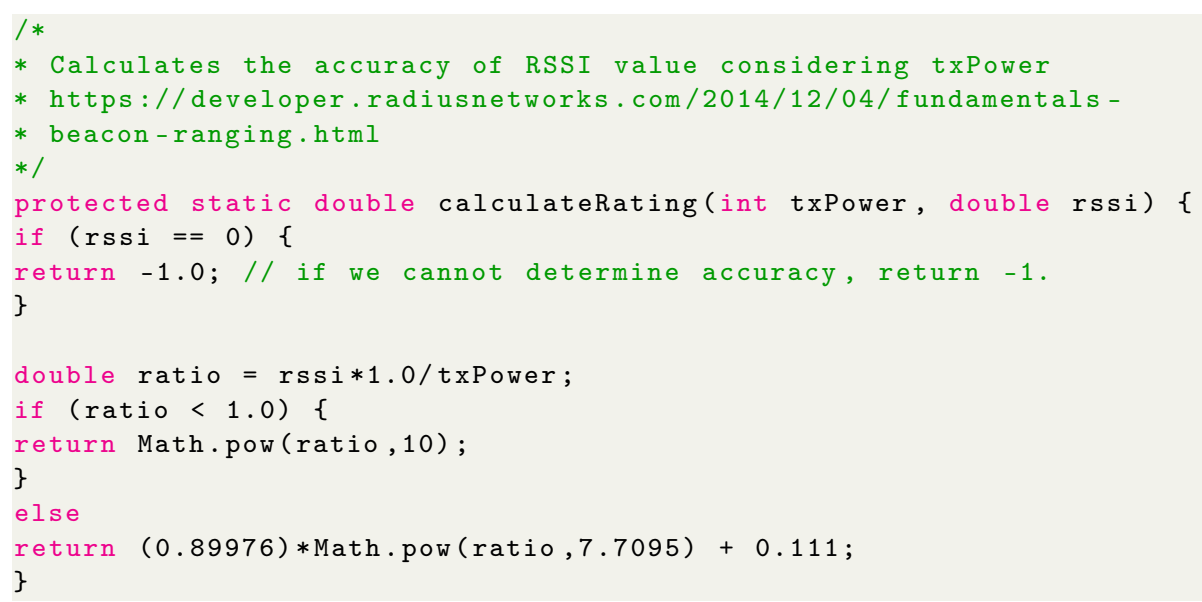

This allows an "accuracy" value, called a rating, to be assigned to each beacon, which is used to correct the detected average RSSI value.

The formula used in the previous code to calculate the rating was [44]:

$$
\text { rating }=0.89976 \cdot\left(\frac{\text { rssi }}{\text { txPower }}\right)^{7.7095}+0.111
$$

The three constants in the formula $(0.89976,7.7095$ and 0.111$)$ are based on a best fit curve based on a number of measured signal strengths at various known distances from a Nexus 4 . However, because the accuracy of this measurement is affected by errors, our algorithm uses the formula generically as a "rating" rather than as a true distance.

Additionally, each beacon is linked to an indoor location (room) via the function in Listing 2. 
Listing 2. Function used to link each beacon to the corresponding room.

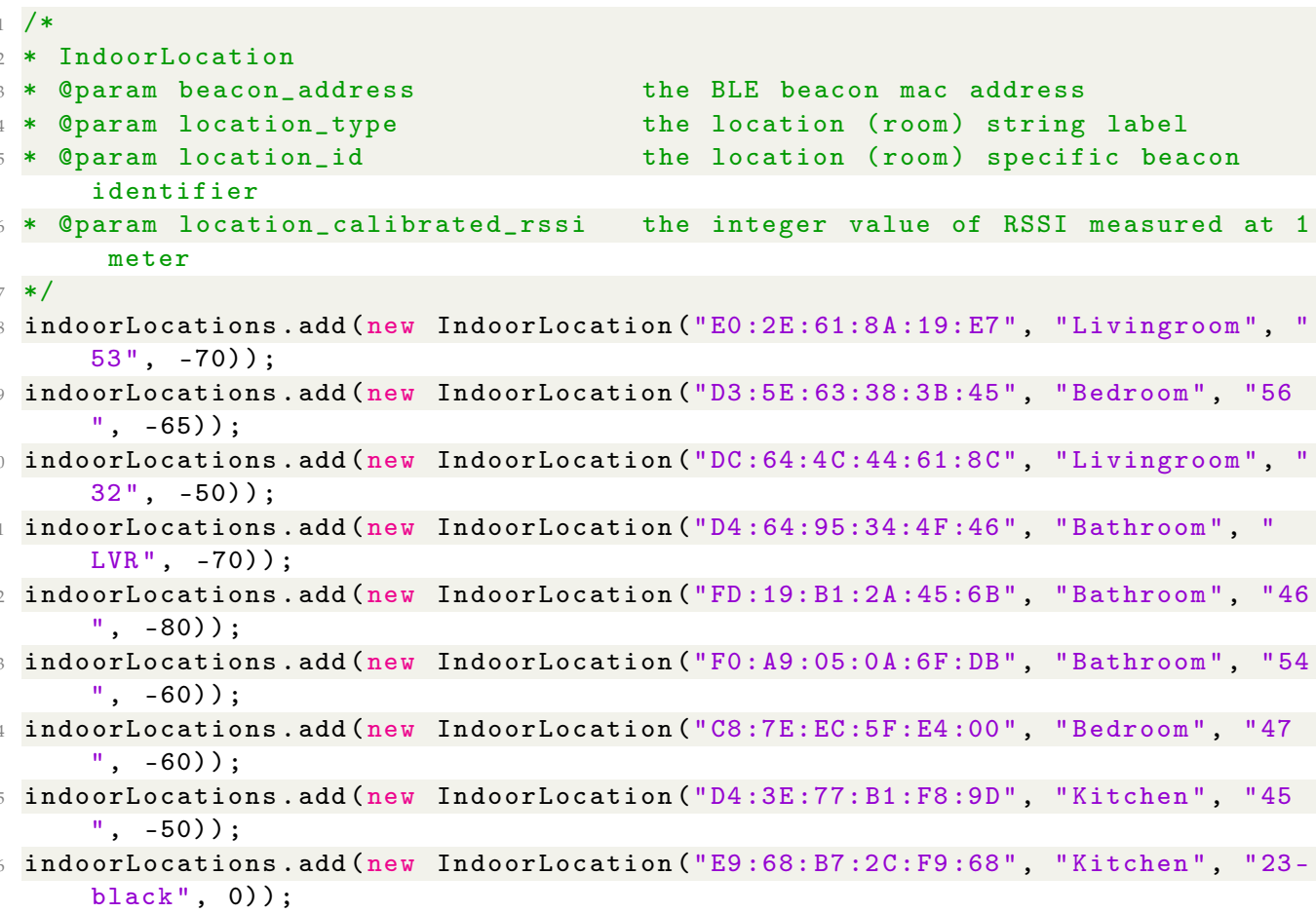

As a result, each beacon is identified by its MAC address, associated with the corresponding room (e.g., living room, bathroom, bedroom or kitchen), and labeled with a location ID label $(53,56,32$, LVR, etc.) In addition, each beacon has a calibration RSSI value that corresponds to the average RSSI value measured at a 1 meter distance.

Finally, the distance from each beacon is calculated using this calibration value and the "log-distance path loss" [45] as reported in Listing 3.

Listing 3. Distance calculation.

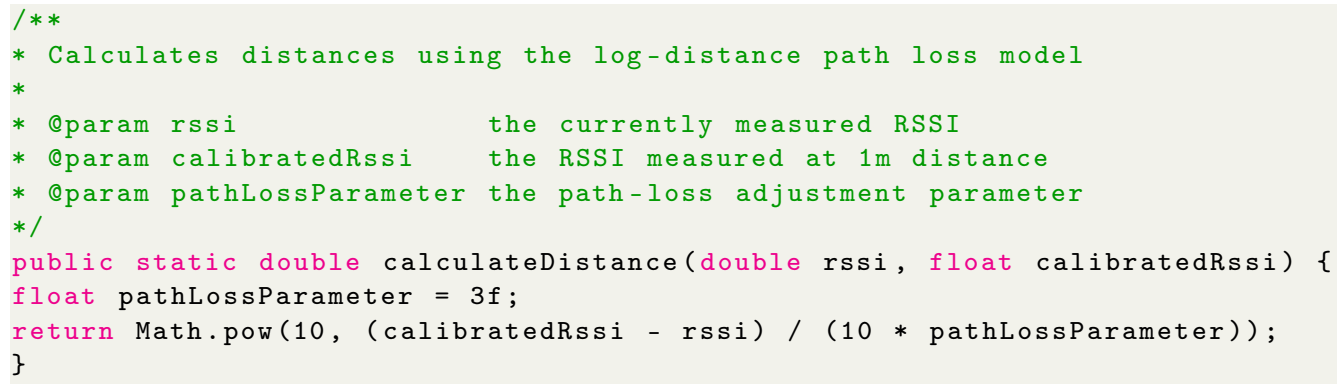

Then, the beacon list is sorted according to the rating value. Since the calculated rating is proportional to the distance, as specified in the formula (1), the first beacon in the list is the beacon with the lowest rating and closest to the smartphone.

The information about the nearest beacon is sent to the cloud server by the application. All detected locations are saved on the server and provided to the location prediction system to be processed, as described in Section 4 .

\section{Location Prediction System}

The location prediction system is based in our previous work $[38,39]$ focused on predicting users' behavior. The algorithm we present in this paper models the user's movements through indoor locations; it uses the semantic location to model them. One of the characteristics of our algorithm is that it works in the semantic-location space instead of the sensor space, which allows us to abstract from the underlying indoor location 
technologies. The location prediction is divided into four modules that process the data sequentially (see Figure 2):

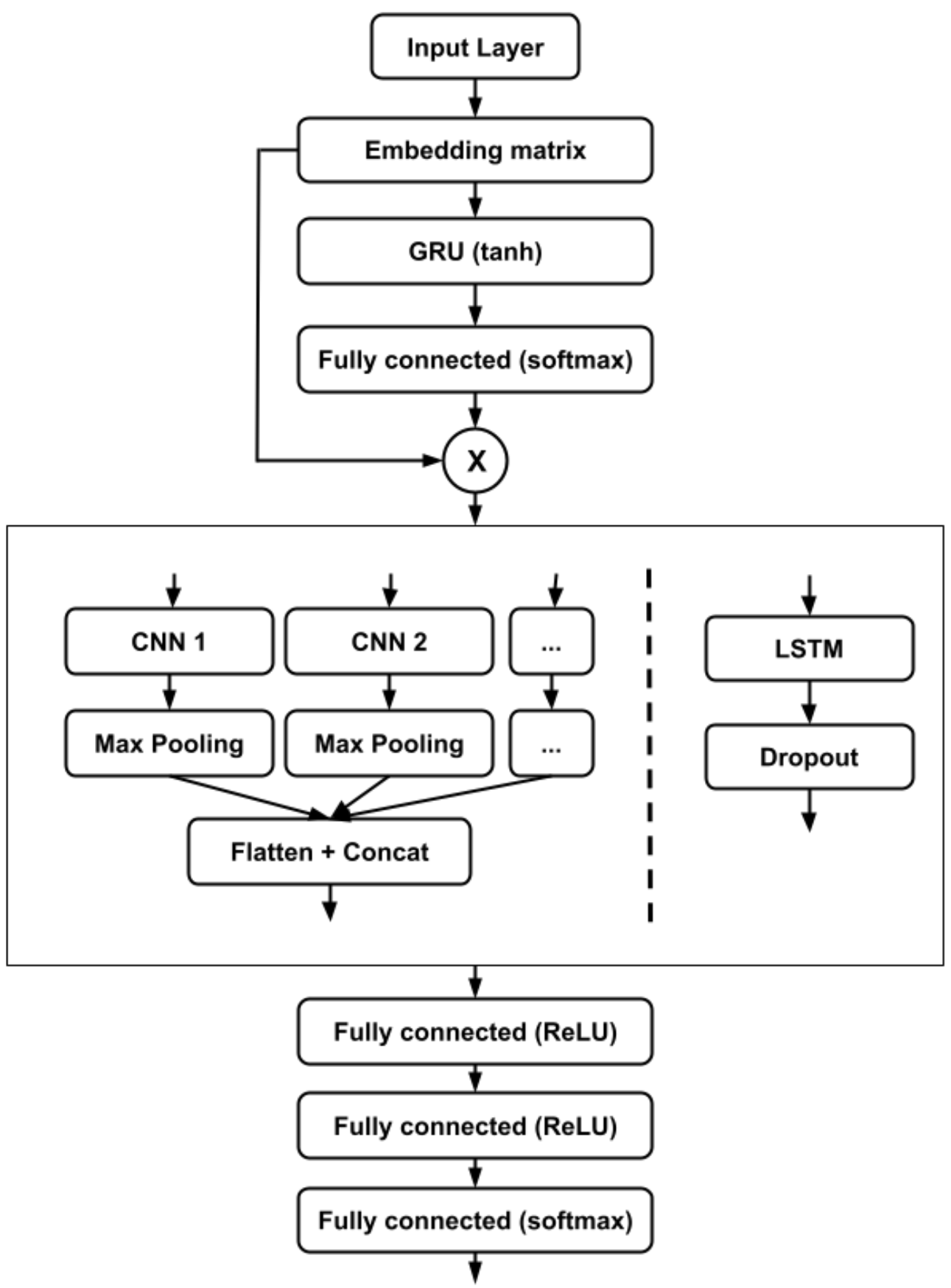

Figure 2. The architecture of the location prediction algorithm. Both approaches are shown in the same image, as they share the input, attention and output modules.

1. Input module: It takes the semantic locations as inputs and transforms them into embeddings to be processed. It has both an input and an embedding layer.

2. Attention mechanism: It evaluates the location embedding sequence to identify those that are more relevant for the prediction process. To do so, it uses a GRU layer, followed by a dense layer with a tanh activation and finally a dense layer with a softmax activation.

3. Sequence feature extractor: It receives the location embeddings processed by the attention mechanism and uses a 1D CNN or a LSTM to identify the most relevelant 
location n-grams of sequences of locations for the prediction. In case of the CNNs, multiple 1D convolution operations are done in parallel to extract n-grams of different lengths in order to obtain a rich representation of the relevant features.

4. Location prediction module: It receives the features extracted by the sequence feature extractor (multi-scale CNNs or LSTMs) and uses those features to predict the next location. This module is composed of two dense layers with ReLU activations and an output dense layer with a softmax activation.

\subsection{Input Module}

The input module is in charge of receiving the location IDs and using the embedding matrix to get the vectors that represent them. As we demonstrated in [38], using better representations, such as embeddings, instead of IDs, provides better predictions. The proposed system uses Word2Vec to obtain the embedding vectors [46], a model widely used in the NLP community.

Given a sequence of locations $S_{l o c}=\left[l_{1}, l_{2}, \ldots, l_{n}\right]$ where $n$ is the length of the sequence and $a_{i} \in \mathbb{R}^{n}$ indicates the location vector of the $i$ th location in the sequence, and Context $\left(l_{i}\right)=\left[l_{i-n}, \ldots, l_{i-1}, l_{i+1}, \ldots, l_{i+n}\right]$ represents the context of $l_{i}$, the window size being $2 n$. $p\left(l_{i} \mid\right.$ Context $\left.\left(l_{i}\right)\right)$ is the probability of $l_{i}$ being in that position of the location instance sequence. To calculate the embeddings, we try to optimize the log maximum likelihood estimation:

$$
L_{l}(M L E)=\sum_{l_{i} \in l_{a c t}} \log p\left(l_{i} \mid \operatorname{Context}\left(l_{i}\right)\right)
$$

Our system uses Gensim to calculate the embedding vectors for each location in the dataset. The location embedding vectors have a size of 50 . To translate the one-hot encoded location IDs to embeddings, we use an embedding matrix, instead of providing the embedding values directly. This allows us to train this matrix and adapt the calculated embeddings to the task at hand, thereby improving the results.

\subsection{Attention Mechanism}

Once we have the semantic embeddings for each location, they are processed by the attention mechanism to identify those locations in the sequence that are more relevant for the prediction process. To do so we use a soft attention mechanism. This is a similar approach to the ones used in NLP to identify the most relevant words in a phrase. However, we do something different in this approach: applying the attention mechanism to the embeddings instead of the hidden steps of the sequence encoder. As proven in [38], this approach has achieved better results when predicting locations.

Location sequences $S_{l o c}$ are temporally ordered sets of locations $l_{t}$, given $t \in[1, T]$. The location sequence $l_{t}, t \in[1 T]$ goes through the input module, which uses the matrix $L_{e}$, calculated previously with Word2Vec, to obtain the location embedding vectors. Those embedding vectors are then processed by the gated recurrent unit layer, creating a representation of the sequence. This gated recurrent unit layer reads the location sequence from $l_{1}$ to $l_{T}$. The used gated recurrent unit layer has a total of 128 units.

$$
\begin{gathered}
x_{t}=L_{e} l_{t} \\
h_{t}=\overrightarrow{\operatorname{GRU}}\left(x_{t}\right)
\end{gathered}
$$

The attention module gets the gated recurrent unit's layer states $h_{t}$ and creates a vector of weights $\alpha_{t} \in[0,1]$ with the relevance of each location $l_{t}$. This is done using a dense layer with a unit size of 128 to get the hidden representation $u_{t}$ of $h_{t}$ :

$$
u_{t}=\tanh \left(W_{l} h_{t}+b_{l}\right)
$$


Then we use a softmax function to calculate the normalized relevance of the weights $\left(\alpha_{t}\right)$ for the location instances:

$$
\alpha_{t}=\frac{\exp \left(u_{t}^{\top} u_{l}\right)}{\sum_{t} \exp \left(u_{t}^{\top} u_{l}\right)}
$$

The obtained vector is used to calculate the relevance of the location embeddings $x_{t}$ for the prediction, $L_{e a d j}$ :

$$
L_{\text {eadj }}=\alpha_{t} x_{t}
$$

Those embeddings $L_{e a d j}$ are the used to process the sequence.

\subsection{Sequence Feature Extractor}

After obtaining the attention modified location embeddings $L_{\text {eadj }}$ in Equation (7), we tested two different approaches to perform the feature extraction: CNNs and LSTMs.

On the one hand, the $\mathrm{CNN}$ architecture was used to extract the features of the sequence. This architecture was composed of multiple 1D CNNs that processed the sequence in parallel with different kernel sizes. This was done to identify differently sized n-grams in the location sequences. The location sequences had a set length: $L_{e a d j}=\left\{l e_{1}, \ldots, l e_{l c}\right\}$. The size of the location embedding was represented by $d_{l e}$, and the elements of the embedding were real numbers, $l e_{i} \in \Re^{d_{l e}}$. After getting the attention modified location embeddings, each location sequence was represented like $L_{e a d j} \in \Re^{l_{l e} \times d_{l e}}$. The convolution operation was:

$$
O_{j}=f\left(W_{j} \circ\left[l e_{1}, \ldots, l e_{l e}-s+1\right]+b\right)
$$

The result of the operation was $O_{j} \in \Re^{l_{l e}-s+1}$, and $W_{j} \in \Re^{l \times d}$, and $b$ were the trained parameters. The activation function $f()$ was a rectified linear unit, and $W \circ L_{\text {eadj }}$ represents the element-wise multiplication. Using $d_{o}$ filter maps, the output of the operation was $O=\left[O_{1}, \ldots, O_{d_{0}}\right] \in \Re^{\left(l_{l e}-s+1\right) \times d_{o}}$. Two hundred filters were used in this case for each convolution operation. After each convolution layer we used 1 max pooling layer. Finally, the results of all the parallel convolution layers were concatenated and flattened.

On the other hand, the LSTM with 512 units received the location embeddings and analysed the existing temporal relations among the different locations that formed each of the sequences. Then, dropout normalization was applied to the extracted features.

\subsection{Location Prediction Module}

The input for the location prediction module is the output of the previously described feature extractor module. To predict the most probable location, this module uses three dense layers. The first two ones $\left(f_{r e}\right)$ use rectified linear units as their activation:

$$
f_{r e}=\operatorname{relu}(W X+b)
$$

To predict the location, the final dense layer uses a softmax activation. The output of this module is a vector with the probabilities of each possible location.

\section{Test Environment}

\subsection{Physical Location}

To assess the indoor positioning system's performance, a realistic scenario was created in which the user wore a smartwatch or placed a smartphone in his or her pocket and moved around his or her home. The house measured $15 \mathrm{~m} \times 7 \mathrm{~m}$ and had five rooms. A path was defined inside the house that led to four different rooms, each with one or more BLE beacon. The user started to follow the established path from the bedroom, as shown in Figure 3. One or more checkpoints were established for each room (indicated by the circle icon).

Three tests have been carried out, each being characterized by different BLE beacon infrastructure, and each test was repeated eight times. Both monitoring devices (smart- 
phone and smartwatch) were used at the same time in each test to compare smartphone and smartwatch performance. The results of the indoor tracking method were read three times at each checkpoint, with a ten-second interval between each detection. The number of false positives was counted for each detection (a false positive occurred when the beacon detected by the mobile application was different from the beacon associated with the checkpoint).

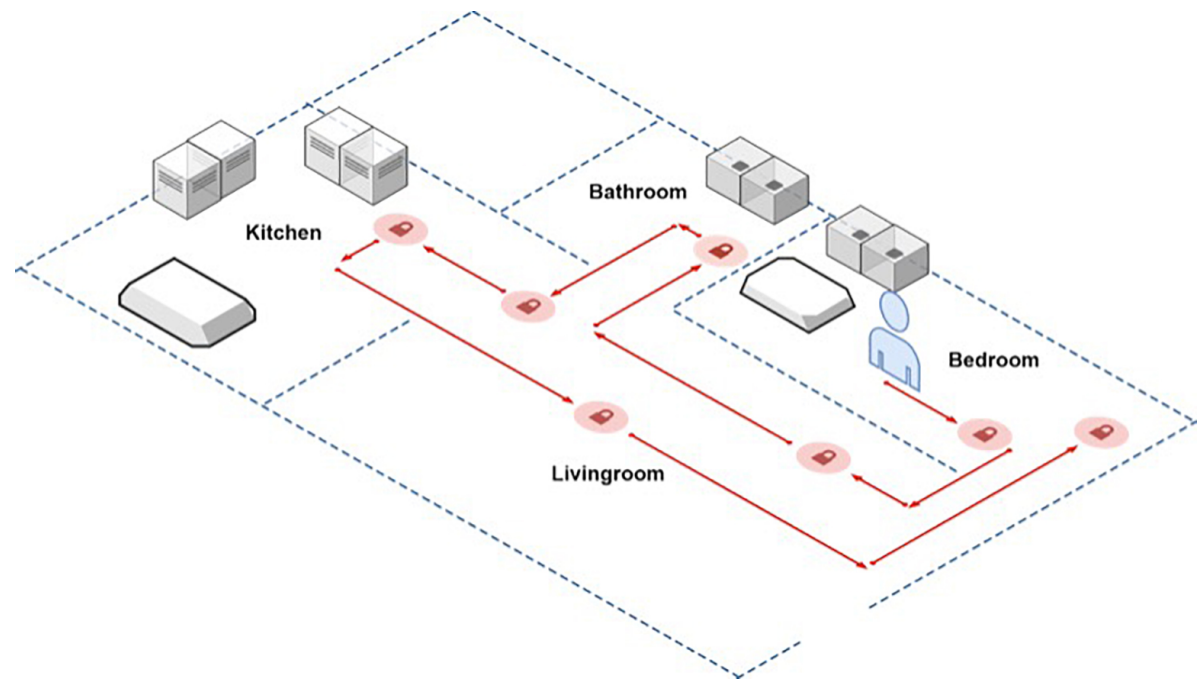

Figure 3. The test environment with the established path and checkpoints.

The configuration used in each test was as follows:

1. Test 1

Beacon model: the battery powered BlueBeacon Mini by BlueUp [47].

Number of beacons: one BLE beacon in each room.

\section{Test 2}

Beacons model: the AKMW-iB005N-SMA by AnkhMaway [48] with a USB power supply. Number of beacons: one BLE beacon in each room.

\section{Test 3}

Beacons models: BlueBeacon Mini and AKMW-iB005N-SMA.

Numbers of beacons: one AKMW-iB005N-SMA in the bedroom, one AKMW-iB005NSMA in the bathroom, two BlueBeacon Mini in the Kitchen and two AKMW-iB005NSMA in the living room.

\subsection{Dataset}

The dataset consists of location data of a single user gathered through a smartwatch over the course of a week. Each time a location change was detected by the smartwatch, the new location and timestamp were stored. In total, the dataset has 267 location changes and four different locations: bedroom, living room, bathroom and kitchen. For the training process, we split the dataset into a training set ( $80 \%$ of the dataset) and a validation set ( $20 \%$ of the dataset) of continuous days.

Since the model uses $n$ previous locations ( 5 in this case) as input to predict the next location, the dataset was split into sequences of $n$ locations, the next location being the one that the model has to predict. Therefore, the training set had 209 training samples, and there were 52 test samples. 


\section{Results}

\subsection{Indoor Location System}

To evaluate the performance of the smartphone and the smartwatch in our indoor location system, the positions detected by the two monitoring devices during the performed tests were compared. For each test and for each iteration, the numbers of false positives at the checkpoints have been calculated. The percentage of error was calculated by the ratio between the number of total false positives and the number of total detections (21 total detections for each test repetition) by using the following equation:

$$
\text { err }_{\text {perc }}=100 \cdot \frac{1}{n} \cdot \sum_{i=1}^{n} x_{i}
$$

where $n$ is the number of total detections and $x_{i}$ is the number of false positives at the $\mathrm{i}$-th iteration (this value was 0 or 1 ). Moreover, for each test and for each iteration, the average absolute deviation (AAD) has been calculated by using the following equation:

$$
A A D=\frac{1}{k} \cdot \sum_{i=1}^{n}\left|y_{i}-\mu\right|
$$

where $y_{i}$ is the number of false positives at the $\mathrm{i}$-th iteration, $\mu$ is the average value of false positives and $k$ is the total number of test repetitions (in our case, eight is the value to assign to the parameter $k$ ).

Table 1 presents the results of test 1 . From this table, it is possible to notice that in this configuration the smartwatch ensured better performance (the mean percentage error was less compared to the mean percentage error obtained with the smartphone as a monitoring device).

\begin{tabular}{|c|c|c|c|c|c|c|}
\hline Test Repetition & $\begin{array}{c}\text { Smartphone } \\
\text { False Positives }\end{array}$ & $\begin{array}{c}\text { Smartphone } \\
\text { Percentage } \\
\text { Error }\end{array}$ & $\begin{array}{c}\text { Smartphone } \\
\text { Absolute } \\
\text { Deviation }\end{array}$ & $\begin{array}{c}\text { Smartwatch } \\
\text { False Positives }\end{array}$ & $\begin{array}{c}\text { Smartwatch } \\
\text { Percentage } \\
\text { Error }\end{array}$ & $\begin{array}{c}\text { Smartwatch } \\
\text { Absolute } \\
\text { Deviation }\end{array}$ \\
\hline 1 & 6 & $28.57 \%$ & 1.625 & 4 & $19.05 \%$ & 0.5 \\
\hline 2 & 6 & $28.57 \%$ & 1.625 & 4 & $19.05 \%$ & 0.5 \\
\hline 3 & 8 & $38.10 \%$ & 0.375 & 6 & $28.57 \%$ & 1.5 \\
\hline 4 & 7 & $33.33 \%$ & 0.625 & 4 & $19.05 \%$ & 0.5 \\
\hline 5 & 9 & $42.86 \%$ & 1.375 & 2 & $9.52 \%$ & 2.5 \\
\hline 6 & 10 & $47.62 \%$ & 2.375 & 6 & $28.57 \%$ & 1.5 \\
\hline 7 & 7 & $33.33 \%$ & 0.625 & 3 & $14.29 \%$ & 1.5 \\
\hline 8 & 8 & $38.10 \%$ & 0.375 & 7 & $33.33 \%$ & 2.5 \\
\hline Average & 7.625 & $36.31 \%$ & 1.125 & 5 & $21.43 \%$ & 1.375 \\
\hline
\end{tabular}

Table 1. Test 1. Percentage error and false positives.

Table 2 presents the results of test 2. In this case, a general improvement of the indoor localization method performance can be observed. The mean percentage error was reduced from $36.31 \%$ to $23.21 \%$ using the smartphone, and from $21.43 \%$ to $13.69 \%$ using the smartwatch. This is because the beacons used in this test had a wider transmission range (the maximum distance at which beacon's signal can be received). In fact, though the transmission range depends of many factors (beacon installation position, operating environment and receiver performance, just to name a few), at the same TxPower of +4 $\mathrm{dBm}$, the theoretical maximum distance (in Line of Sight free-space condition) offered by the AKMW-iB005N-SMA is $130 \mathrm{~m}$, which is $100 \mathrm{~m}$ greater than for the BlueBeacon Mini. Additionally, in this configuration, the performance registered using the smartwatch as the monitoring device was better. 
Table 2. Test 2. Percentage error and false positives.

\begin{tabular}{|c|c|c|c|c|c|c|}
\hline Test Repetition & $\begin{array}{c}\text { Smartphone } \\
\text { False Positives }\end{array}$ & $\begin{array}{c}\text { Smartphone } \\
\text { Percentage } \\
\text { Error }\end{array}$ & $\begin{array}{c}\text { Smartphone } \\
\text { Absolute } \\
\text { Deviation }\end{array}$ & $\begin{array}{c}\text { Smartwatch } \\
\text { False Positives }\end{array}$ & $\begin{array}{c}\text { Smartwatch } \\
\text { Percentage } \\
\text { Error }\end{array}$ & $\begin{array}{c}\text { Smartwatch } \\
\text { Absolute } \\
\text { Deviation }\end{array}$ \\
\hline 1 & 9 & $42.86 \%$ & 4.125 & 3 & $14.29 \%$ & 0.125 \\
\hline 2 & 6 & $28.57 \%$ & 1.125 & 3 & $14.29 \%$ & 0.125 \\
\hline 3 & 4 & $19.05 \%$ & 0.875 & 3 & $14.29 \%$ & 1.125 \\
\hline 4 & 4 & $19.05 \%$ & 0.875 & 1 & $4.76 \%$ & 1.875 \\
\hline 5 & 3 & $14.29 \%$ & 1.875 & 2 & $9.52 \%$ & 0.875 \\
\hline 6 & 4 & $19.05 \%$ & 0.875 & 2 & $9.52 \%$ & 0.875 \\
\hline 7 & 9 & $42.86 \%$ & 4.125 & 5 & $23.81 \%$ & 2.125 \\
\hline 8 & 0 & $0.00 \%$ & 4.875 & 4 & $19.05 \%$ & 1.125 \\
\hline Average & 4.875 & $23.21 \%$ & 2.344 & 3 & $13.69 \%$ & 0.906 \\
\hline
\end{tabular}

Finally, Table 3 presents the results of the test 3 . In this test the mean percentage error using the smartphone was higher compared to the mean percentage error obtained in test 2 for the same monitoring device. This was due to the high reception capacity of the smartphone antenna, which was too sensitive if several beacons were placed close in the same environment. The mean percentage error using the smartwatch was reduced to $7.74 \%$. It is possible to draw some conclusions based on the results of all three tests. Several factors, such as the positions of the beacons within the room (for example, height from the ground) or the distances between the beacons, can influence indoor tracking results. For example, beacons close to each other can cause interference. However, in general, the smartwatch guaranteed better performance in indoor localization than the smartphone with the same configuration in BLE beacon infrastructure.

Table 3. Test 3. Percentage error and false positives.

\begin{tabular}{|c|c|c|c|c|c|c|}
\hline Test Repetition & $\begin{array}{c}\text { Smartphone } \\
\text { False Positives }\end{array}$ & $\begin{array}{l}\text { Smartphone } \\
\text { Percentage } \\
\text { Error }\end{array}$ & $\begin{array}{c}\text { Smartphone } \\
\text { Absolute } \\
\text { Deviation }\end{array}$ & $\begin{array}{c}\text { Smartwatch } \\
\text { False Positives }\end{array}$ & $\begin{array}{c}\text { Smartwatch } \\
\text { Percentage } \\
\text { Error }\end{array}$ & $\begin{array}{c}\text { Smartwatch } \\
\text { Absolute } \\
\text { Deviation }\end{array}$ \\
\hline 1 & 6 & $28.57 \%$ & 0.25 & 3 & $14.29 \%$ & 1.375 \\
\hline 2 & 9 & $42.86 \%$ & 3.25 & 1 & $4.76 \%$ & 0.625 \\
\hline 3 & 5 & $23.81 \%$ & 0.75 & 2 & $9.52 \%$ & 0.375 \\
\hline 4 & 3 & $14.29 \%$ & 2.75 & 3 & $14.29 \%$ & 1.375 \\
\hline 5 & 6 & $28.57 \%$ & 0.25 & 2 & $9.52 \%$ & 0.375 \\
\hline 6 & 6 & $28.57 \%$ & 0.25 & 0 & $0.00 \%$ & 1.625 \\
\hline 7 & 5 & $23.81 \%$ & 0.75 & 1 & $4.76 \%$ & 0.625 \\
\hline 8 & 6 & $28.57 \%$ & 0.25 & 1 & $4.76 \%$ & 0.625 \\
\hline Average & 5.75 & $27.38 \%$ & 1.063 & 2 & $7.74 \%$ & 0.875 \\
\hline
\end{tabular}

\subsection{Location Prediction System}

We have evaluated the proposed approach by comparing our results in terms of accuracy score with two different approaches that have been used in the location prediction literature as baselines: nearest locations (NL) where the nearest neighbor to the user's current location is selected [49], and a hidden Markov model which characterizes the movement patterns [50]. As can be seen in Table 4, our approaches outperformed the proposed baselines by a wide margin. 
Moreover, to give more insights into the performance of the proposed architectures, we have evaluated the proposed location prediction system using the top-k accuracy score. This score measures how many times the ground truth (or correct label) is among the top $k$ predicted labels provided by the fully connected layer with a softmax activation function.

Table 4. Accuracy scores of the performed experiments. Nearest locations (NL), hidden Markov models (HMM), multi-scale CNNs (M1), multi-scale CNNs with attention (M2), LSTM (L1), LSTM with attention (L2).

\begin{tabular}{cc}
\hline ID & Accuracy \\
\hline NL & 0.5 \\
HMM & 0.5961 \\
\hline M1 & 0.6538 \\
M2 & 0.6538 \\
\hline L1 & 0.6346 \\
L2 & $\mathbf{0 . 6 7 3 1}$ \\
\hline
\end{tabular}

$l_{i}$ is the correct location, $T_{i}^{k}$ the ordered list of the top $k$ predicted locations, $N$ the number of tests samples and $b$ the scoring function with two possible outputs 0,1 . The top-k accuracy is formulated in Equation (12):

$$
\text { acc_at_k }=\frac{1}{N} \sum_{i=1}^{N} b\left[l_{i} \in T_{i}^{k}\right]
$$

Therefore, the value of the scoring function is 1 when the label of the correct location exists in the ordered list of the top $k$ predicted locations. On the contrary, if the label is not in the ordered list, the function will return 0 . For this experimentation, we report the accuracy scores with $k=1, k=2$ and $k=3$ since we had a total of four possible outcomes.

Table 5 presents the results. The best results for accuracy at 1 and 3 were obtained in experiment L2, where the LSTM with the previously mentioned attention mechanism was used. However, the best metric for accuracy at 2 was obtained in M2, where multi-scale convolutional neural networks were used with the same attention mechanism. In both cases, the best results were achieved using the embedding level attention mechanism introduced in [38]. However, in this dataset the best results overall were achieved using LSTMs.

Table 5. Top-k accuracy score results of the best performing experiments. Multi-scale CNNs (M1), multi-scale CNNs with attention (M2), LSTM (L1), LSTM with attention (L2).

\begin{tabular}{cccc}
\hline ID & acc_at_1 & acc_at_2 & acc_at_3 \\
\hline M1 & 0.6538 & 0.9038 & 0.9423 \\
M2 & 0.6538 & $\mathbf{0 . 9 2 3 1}$ & 0.9423 \\
\hline L1 & 0.6346 & 0.7885 & 0.9423 \\
L2 & $\mathbf{0 . 6 7 3 1}$ & 0.8654 & $\mathbf{0 . 9 8 0 8}$ \\
\hline
\end{tabular}

\section{Conclusions}

In this paper we present an indoor locating system based on BLE and its evaluation utilizing a smartphone and a smartwatch as monitoring devices. Over that system, we built a behavior prediction system based on locations and validated two different approaches. Our system provides a holistic approach to an indoor location system, providing both the necessary infrastructure and the intelligent framework over it.

The system's performance in terms of mean percentage error was assessed and analyzed. A distinct BLE beacon infrastructure was considered for each test by altering the quantity and models of BLE beacons in each room of the considered indoor environment. The best results were achieved using the smartwatch instead of the smartphone. 
Furthermore, a position prediction system based on neural embeddings to represent the locations of a house was introduced, along with an attention-based mechanism that modifies those embeddings rather than having them applied to the hidden states of the neural network design. The location prediction system's accuracy has also been assessed, compared with other approaches and discussed. From these experiments, two main conclusions can be drawn: first, the proposed attention mechanism applied to the embeddings improves the architecture's performance; and second, despite the limited size of training samples, the presented deep neural network architectures performed better than shallow machine learning algorithms such as hidden Markov models.

The system will be expanded in the future by including RFID components, such as wearable RFID devices and RFID tags, to capture data that will be processed by an activity recognition module. From location data and RFID data, this module will be able to deduce user activities. Additionally, as a future extension of the presented work, it would be interesting to use algorithms such as the Kalman filter to improve the results of our indoor location system. Regarding the location prediction system, using the transformers introduced by Vaswani et al. [51] could improve its performance.

Author Contributions: Conceptualization, A.A., A.B.-J., I.S. and L.P.; methodology, A.B.-J., A.A., I.S., T.M., L.F., M.E. and L.P.; software, A.B.-J., A.A., I.S. and L.F.; validation, A.B.-J., A.A., I.S., T.M., L.F., M.E. and L.P.; formal analysis, A.B.-J., A.A., I.S., T.M., L.F., M.E. and L.P.; writing, A.B.-J., I.S., A.A. and L.P.; supervision, A.A. and L.P. All authors have read and agreed to the published version of the manuscript.

Funding: This research was funded by the project FuturAAL-Ego (RTI2018-101045-A-C22) granted by Spanish Ministry of Science, Innovation and Universities; and the project BD4QoL (875192) granted by the European Commission.

Conflicts of Interest: The authors declare no conflict of interest. The funders had no role in the design of the study; in the collection, analyses, or interpretation of data; in the writing of the manuscript, or in the decision to publish the results.

\section{References}

1. Khalaf, R.; Mohammed, A.; Essa, E.; Ali, H. Controlling Smart Home Activities Using IoT. In Proceedings of the 2019 International Conference on Computing and Information Science and Technology and Their Applications(ICCISTA), Kirkuk, Iraq, 3-5 March 2019; IEEE: Piscataway, NJ, USA, 2019; pp. 1-6.

2. Catarinucci, L.; Colella, R.; Consalvo, S.I.; Patrono, L.; Salvatore, A.; Sergi, I. IoT-oriented waste management system based on new RFID-sensing devices and cloud technologies. In Proceedings of the 2019 th International Conference on Smart and Sustainable Technologies(SpliTech), Split, Croatia, 18-21 June 2019; IEEE: Piscataway, NJ, USA, 2019; pp. 1-5.

3. Sánchez-Corcuera, R.; Nuñez-Marcos, A.; Sesma-Solance, J.; Bilbao-Jayo, A.; Mulero, R.; Zulaika, U.; Azkune, G.; Almeida, A. Smart cities survey: Technologies, application domains and challenges for the cities of the future. Int. J. Distrib. Sens. Netw. 2019, 15. [CrossRef]

4. Mulero, R.; Almeida, A.; Azkune, G.; Abril-Jiménez, P.; Waldmeyer, M.T.A.; Castrillo, M.P.; Patrono, L.; Rametta, P.; Sergi, I. An IoT-aware approach for elderly-friendly cities. IEEE Access 2018, 6, 7941-7957. [CrossRef]

5. Maghdid, S.A.; Maghdid, H.S.; Rahman, S. Tracking Indoor Elderly-People Using Onboard Smartphones Wi-Fi Device and Inertial Sensors. In Proceedings of the 2018 1st International Conference on Advanced Research in Engineering Sciences (ARES), Dubai, United Arab Emirates, 15 June 2018; IEEE: Piscataway, NJ, USA, 2018; pp. 1-8.

6. López-de Ipiña, D.; Lorido, T.; López, U. Indoor navigation and product recognition for blind people assisted shopping In Proceedings of the International Workshop on Ambient Assisted Living, Torremolinos-Malaga, Spain, 8-10 June 2011; Springer: Berlin, Germany, 2011; pp. 33-40.

7. Nakashima, K.; Okamoto, E. An energy-efficient adaptive lighting control scheme using indoor localization with prior position information. In Proceedings of the 2015 7th International Conference on Information Technology and Electrical Engineering (ICITEE), Chiang Mai, Thailand, 29-30 October 2015; IEEE: Piscataway, NJ, USA, 2015; pp. 470-475.

8. Umer, S.M.; Ou, Y.; Feng, W. A novel localization and navigation approach for an indoor autonomous mobile surveillance robot. In Proceedings of the 2014 4th IEEE International Conference on Information Science and Technology, Shenzhen, China, 26-28 April 2014; IEEE: Piscataway, NJ, USA, 2014; pp. 5-10.

9. Almeida, A.; Fiore, A.; Mainetti, L.; Mulero, R.; Patrono, L.; Rametta, P. An IoT-aware architecture for collecting and managing data related to elderly behavior. Wirel. Commun. Mob. Comput. 2017, 2017, 5051915. [CrossRef] 
10. Alletto, S.; Cucchiara, R.; Del Fiore, G.; Mainetti, L.; Mighali, V.; Patrono, L.; Serra, G. An indoor location-aware system for an IoT-based smart museum. IEEE Internet Things J. 2015, 3, 244-253. [CrossRef]

11. Eksen, K.; Serif, T.; Ghinea, G.; Grønli, T.M. Inloc: Location-aware emergency evacuation assistant. In Proceedings of the 2016 IEEE International Conference on Computer and Information Technology (CIT), Nadi, Fiji, 8-10 December 2016; IEEE: Piscataway, NJ, USA, 2016; pp. 50-56.

12. Do, T.H.; Yoo, M. An in-depth survey of visible light communication based positioning systems. Sensors 2016, 16, 678. [CrossRef] [PubMed]

13. Want, R.; Hopper, A.; Falcao, V.; Gibbons, J. The active badge location system. ACM Trans. Inf. Syst. (TOIS) 1992, 10, 91-102. [CrossRef]

14. Lo, D.; Mendonça, P.R.; Hopper, A. TRIP: A low-cost vision-based location system for ubiquitous computing. Pers. Ubiquitous Comput. 2002, 6, 206-219.

15. Zhou, Z.; Chen, X.; Chung, Y.C.; He, Z.; Han, T.X.; Keller, J.M. Video-based activity monitoring for indoor environments. In Proceedings of the 2009 IEEE International Symposium on Circuits and Systems, Taipei, Taiwan, 24-27 May 2009; IEEE: Piscataway, NJ, USA, 2009; pp. 1449-1452.

16. Torres-Sospedra, J.; Montoliu, R.; Trilles, S.; Belmonte, Ó.; Huerta, J. Comprehensive analysis of distance and similarity measures for Wi-Fi fingerprinting indoor positioning systems. Expert Syst. Appl. 2015, 42, 9263-9278. [CrossRef]

17. Catarinucci, L.; Colella, R.; De Blasi, M.; Mighali, V.; Patrono, L.; Tarricone, L. High performance RFID tags for item-level tracing systems. In Proceedings of the SoftCOM 2010, 18th International Conference on Software, Telecommunications and Computer Networks, Split, Dubrovnik, Croatia, 23-25 September 2010; IEEE: Piscataway, NJ, USA, 2010; pp. 21-26.

18. Liu, X.; Peng, J.; Liu, T. A novel indoor localization system based on passive RFID technology. In Proceedings of the 2011 International Conference on Electronic \& Mechanical Engineering and Information Technology, Harbin, China, 12-14 August 2011; IEEE: Piscataway, NJ, USA, 2011; Volume 8, pp. 4285-4288.

19. Xu, H.; Ding, Y.; Li, P.; Wang, R.; Li, Y. An RFID indoor positioning algorithm based on Bayesian probability and K-nearest neighbor. Sensors 2017, 17, 1806. [CrossRef]

20. Jiang, H.; Peng, C.; Sun, J. Deep belief network for fingerprinting-based RFID indoor localization. In Proceedings of the 2019 IEEE International Conference on Communications (ICC), Shanghai, China, 22-24 May 2019; IEEE: Piscataway, NJ, USA, 2019; pp. 1-5.

21. Zeng, Y.; Chen, X.; Li, R.; Tan, H.Z. UHF RFID indoor positioning system with phase interference model based on double tag array. IEEE Access 2019, 7, 76768-76778. [CrossRef]

22. Sakpere, W.; Adeyeye-Oshin, M.; Mlitwa, N.B. A state-of-the-art survey of indoor positioning and navigation systems and technologies. S. Afr. Comput. J. 2017, 29, 145-197. [CrossRef]

23. Catarinucci, L.; Colella, R.; Mainetti, L.; Mighali, V.; Patrono, L.; Sergi, I.; Tarricone, L. Performance evaluation of a novel animals tracking system based on UHF RFID technology. J. Commun. Softw. Syst. 2013, 9, 4-13. [CrossRef]

24. Kajioka, S.; Mori, T.; Uchiya, T.; Takumi, I.; Matsuo, H. Experiment of indoor position presumption based on RSSI of Bluetooth LE beacon. In Proceedings of the 2014 IEEE 3rd Global Conference on Consumer Electronics (GCCE), Tokyo, Japan, 7-10 October 2014; IEEE: Piscataway, NJ, USA, 2014; pp. 337-339.

25. Faragher, R.; Harle, R. An analysis of the accuracy of bluetooth low energy for indoor positioning applications. In Proceedings of the 27th International Technical Meeting of The Satellite Division of the Institute of Navigation (ION GNSS + 2014), Tampa, FL, USA, 8-12 September 2014; ION: Leesburg, VA, USA, 2014; pp. 201-210.

26. Subedi, S.; Kwon, G.R.; Shin, S.; Hwang, S.s.; Pyun, J.Y. Beacon based indoor positioning system using weighted centroid localization approach. In Proceedings of the 2016 Eighth International Conference on Ubiquitous and Future Networks (ICUFN), Vienna, Austria, 5-8 July 2016; IEEE: Piscataway, NJ, USA, 2016; pp. 1016-1019.

27. Ji, M.; Kim, J.; Jeon, J.; Cho, Y. Analysis of positioning accuracy corresponding to the number of BLE beacons in indoor positioning system. In Proceedings of the 2015 17th International Conference on Advanced Communication Technology (ICACT), PyeongChang, Korea, 1-3 July 2015; IEEE: Piscataway, NJ, USA, 2015; pp. 92-95.

28. García, E.; Poudereux, P.; Hernández, Á.; Ureña, J.; Gualda, D. A robust UWB indoor positioning system for highly complex environments. In Proceedings of the 2015 IEEE International Conference on Industrial Technology (ICIT), Seville, Spain, 17-19 March 2015; IEEE: Piscataway, NJ, USA, 2015; pp. 3386-3391.

29. Minne, K.; Macoir, N.; Rossey, J.; Van den Brande, Q.; Lemey, S.; Hoebeke, J.; De Poorter, E. Experimental evaluation of UWB indoor positioning for indoor track cycling. Sensors 2019, 19, 2041. [CrossRef] [PubMed]

30. Ridolfi, M.; Vandermeeren, S.; Defraye, J.; Steendam, H.; Gerlo, J.; De Clercq, D.; Hoebeke, J.; De Poorter, E. Experimental evaluation of UWB indoor positioning for sport postures. Sensors 2018, 18, 168. [CrossRef] [PubMed]

31. Cadena, C.; Carlone, L.; Carrillo, H.; Latif, Y.; Scaramuzza, D.; Neira, J.; Reid, I.; Leonard, J.J. Past, present, and future of simultaneous localization and mapping: Toward the robust-perception age. IEEE Trans. Robot. 2016, 32, 1309-1332. [CrossRef]

32. Mainetti, L.; Patrono, L.; Sergi, I. A survey on indoor positioning systems. In Proceedings of the 2014 22nd International Conference on Software, Telecommunications and Computer Networks (SoftCOM), Split, Croatia, 17-19 September 2014; IEEE: Piscataway, NJ, USA, 2014; pp. 111-120.

33. Irizar-Arrieta, A.; Gómez-Carmona, O.; Bilbao-Jayo, A.; Casado-Mansilla, D.; Lopez-De-Ipina, D.; Almeida, A. Addressing behavioural technologies through the human factor: A review. IEEE Access 2020, 8, 52306-52322. [CrossRef] 
34. Deshpande, D.; Deshpande, S. Analysis of various characteristics of online user behavior models. Int. J. Comput. Appl. 2017, 161, 5-10. [CrossRef]

35. Raju, S.S.; Dhandayudam, P. Prediction of customer behaviour analysis using classification algorithms. In Proceedings of the AIP Conference, Maharashtra, India, 5-6 July 2018; AIP Publishing LLC: New York, NY, USA, 2018; Volume 1952, p. 020098.

36. Zhan, Y.; Haddadi, H. Towards automating smart homes: Contextual and temporal dynamics of activity prediction. In Proceedings of the 2019 ACM International Joint Conference on Pervasive and Ubiquitous Computing and Proceedings of the 2019 ACM International Symposium on Wearable Computers, London, UK, 9-13 September 2019; ACM: New York, USA, 2019; pp. 413-417.

37. Köse, A.; Tepljakov, A.; Petlenkov, E. Dynamic predictive modeling approach of user behavior in virtual reality based application. In Proceedings of the 2019 27th Mediterranean Conference on Control and Automation (MED), Akko, Israel, 1-4 July 2019; IEEE: Piscataway, NJ, USA, 2019; pp. 57-62.

38. Almeida, A.; Azkune, G.; Bilbao, A. Embedding-level attention and multi-scale convolutional neural networks for behaviour modelling. In Proceedings of the 2018 IEEE SmartWorld, Ubiquitous Intelligence \& Computing, Advanced \& Trusted Computing, Scalable Computing \& Communications, Cloud \& Big Data Computing, Internet of People and Smart City Innovation (SmartWorld/SCALCOM/UIC/ATC/CBDCom/IOP/SCI), Guangzhou, China, 8-12 October 2018; IEEE: Piscataway, NJ, USA, 2018; pp. 439-445.

39. Almeida, A.; Azkune, G. Predicting human behaviour with recurrent neural networks. Appl. Sci. 2018, 8, 305. [CrossRef]

40. Pan, Y.; Zhang, L. BIM log mining: Learning and predicting design commands. Autom. Constr. 2020, 112, 103107. [CrossRef]

41. Dash, S.; Luhach, A.K.; Chilamkurti, N.; Baek, S.; Nam, Y. A neuro-fuzzy approach for user behaviour classification and prediction. J. Cloud Comput. 2019, 8, 1-15.

42. Kim, D.; Kim, S.; Zhao, H.; Li, S.; Rossi, R.A.; Koh, E. Domain switch-aware holistic recurrent neural network for modeling multi-domain user behavior. In Proceedings of the Twelfth ACM International Conference on Web Search and Data Mining, Melbourne, Australia, 11-15 February 2019; ACM: New York, NY, USA, 2019; pp. 663-671.

43. Salian, N. Visual Attention and Memory Augmented Activity Recognition and Behavioral Prediction. In Proceedings of the International Conference on Applications and Techniques in Information Security, Nanning, China, 9-11 November 2018; Springer: Berlin, Germany, 2018; pp. 92-106.

44. Young, D.G. The Challenge of Bluetooth Distance Estimation. Available online: https://github.com/davidgyoung/ davidgyoung.github.io/blob/32ba6f44e1af2b5df08589cee286eaf940566ab7/_posts/2020-05-15-how-far-can-you-go.md (accessed on 5 July 2021).

45. Wikipedia. Log-Distance Path Loss Model. Available online: https://en.wikipedia.org/wiki/Log-distance_path_loss_model (accessed on 10 June 2021).

46. Mikolov, T.; Sutskever, I.; Chen, K.; Corrado, G.; Dean, J. Distributed representations of words and phrases and their compositionality. arXiv 2013, arXiv:1310.4546.

47. BlueUp. BlueBeacon Mini. Available online: https://www.blueupbeacons.com/index.php?page=mini (accessed on 10 June 2021).

48. AnkhMaway. AKMW-iB005N-SMA Beacon. Available online: https://www.beaconzone.co.uk/AKMW-iB005N-SMAEddystone (accessed on 10 June 2021).

49. Duong-Trung, N.; Schilling, N.; Schmidt-Thieme, L. Near real-time geolocation prediction in twitter streams via matrix factorization based regression. In Proceedings of the 25th ACM International on Conference on Information and Knowledge Management, Indianapolis, IN, USA, 24-28 October 2016; ACM: New York, NY, USA, 2016; pp. $1973-1976$.

50. Mathew, W.; Raposo, R.; Martins, B. Predicting future locations with hidden Markov models. In Proceedings of the 2012 ACM Conference on Ubiquitous Computing, Pittsburgh, PA, USA, 5-8 September 2012; ACM: New York, NY, USA, 2012; pp. 911-918.

51. Vaswani, A.; Shazeer, N.; Parmar, N.; Uszkoreit, J.; Jones, L.; Gomez, A.N.; Kaiser, Ł.; Polosukhin, I. Attention is all you need. In Proceedings of the 31st International Conference on Neural Information Processing Systems, Long Beach, CA, USA, 4-9 December 2017; ACM: New York, NY, USA, 2017; pp. 6000-6010. 